\title{
Implementación de un Sistema de Gestión de la Calidad en los Laboratorios del CIRA/UNAN
}

Iris Hurtado García.

Centro para la Investigación en Recursos Acuáticos de Nicaragua, Universidad Nacional Autónoma de Nicaragua, Apdo. Postal 4598, Managua, Nicaragua.

Correo electrónico: Iris.hurtado@cira-unan.edu.ni

Recepcionado: 21 Diciembre 2009 / Aceptado: 14 Abril 2010

\section{Resumen}

1 1 Centro para la Investigación en Recursos Acuáticos de Nicaragua (CIRA/UNAN), desde su fundación ha tenido como visión convertirse en un centro de investigación líder a nivel nacional y regional. Actualmente la globalización demanda que los laboratorios de ensayo demuestren competencia técnicas, lo cual se logra a través del establecimiento de un sistema de gestión de la calidad, que mejore continuamente la capacidad operacional y la competencia técnica del laboratorio. En este contexto, surge la necesidad de establecer e implementar un sistema de gestión de la calidad en el CIRA/UNAN, que asegure su competencia técnica, mediante el apego a la norma ISO/IEC 17025:2005 o su equivalente nacional NTN 04 001 05, por ser el mecanismo internacionalmente aceptado para la demostración de la competencia técnica de los laboratorios. La implementación del sistema de gestión de la calidad en los laboratorios del CIRA/UNAN, se desarrolló en cuatro etapas fundamentales, que consistieron en: Diagnostico situacional del Centro, establecimiento del Sistema de Gestión de la Calidad, Implementación y Mantenimiento del mismo. Para alcanzar el éxito en este proceso, fue necesario el involucramiento y la apropiación del sistema de gestión de la calidad por cada uno de los miembros de la organización, así como el compromiso y apoyo de la dirección del Centro. Durante el establecimiento del sistema de gestión de la calidad, fue necesario adecuar la operatividad del centro a los requisitos establecidos en la Norma ISO/IEC 17025:2005, bajo la consideración; que el sistema de gestión debía facilitar la operatividad del Centro. Un aspecto de suma importancia para alcanzar y mantener el éxito en este proceso ha sido la mejora continua del sistema de gestión de la calidad. De manera que el CIRA/UNAN cuenta con un sólido y completo sistema de gestión de calidad que rige todas las operaciones administrativas y técnicas asociadas a la generación de resultados, proyectos, informes e interpretaciones, Así como el logro de la acreditación de nueve ensayos microbiológicos, lo que posiciono al centro como uno de los más prestigioso centros de investigación a nivel nacional.

Palabras claves: Calidad, Acreditación, ISO 17025, laboratorios, CIRA/UNAN 


\section{Abstract}

The Nicaraguan Research Center for Aquatic Resources of Nicaragua (CIRA/UNAN), since its foundation has had the vision to become a national and regional leader in research. Currently, globalization demands that testing laboratories demonstrate technical capacity, usually achieved through the establishment of quality management system, which improves continually the operational capacity and the technical competence of the laboratory. In this context, it was necessary to establish and implement a quality management system at CIRA/ UNAN, to ensure its technical competence, using the international norm, ISO/IEC 17025:2005 or its national equivalent the NTN 04001 05, which is the international mechanism accepted for demonstrating laboratory technical competence. The management quality system in CIRA/UNAN laboratories was developed in four key stages: A diagnostic of the situation in the center, establishment of the Quality Management System, and its implementation and maintenance. To achieve success in this process, it was necessary to promote the involvement and ownership of the system of quality management by each organization's member, as well as the commitment and support from the Center's direction. During the establishing of the quality management system, it was necessary to adjust the center's operational structure to the ISO/IEC 17025:2005 requirements, taking in account this consideration: The management system should facilitate the operation of the Center. One aspect of the utmost importance to achieve and maintain the success in this process has been the continuing improvement of the quality management system. So now CIRA/UNAN has a comprehensive and sound quality management system that governs all administrative and technical operations associated with the generation of results, projects, reports and interpretations. CIRA/UNAN has achieved the accreditation of nine microbiological tests, which situates the center as one of the most prestigious research centers at national level.

\section{Introducción}

La calidad no es un tema nuevo ya que cerca del año 1450 a. C., los inspectores egipcios comprobaban las medidas de los bloques de piedra con un pedazo de cordel. La mayoría de las civilizaciones antiguas daban gran importancia a la equidad en los negocios y cómo resolver las quejas, aún cuando esto implicara condenar al responsable a la muerte. Desde esas épocas a la fecha la calidad ha evolucionado paulatinamente, pasando de los procesos de inspección en el Siglo XIX, a la etapa actual de la administración estratégica de la calidad (década de los 90s), donde se hace hincapié en el mercado y en las necesidades del consumidor, reconociendo el efecto estratégico de la calidad, como una oportunidad de competitividad.

El Centro para la Investigación en Recursos Acuáticos de Nicaragua (CIRA/UNAN), desde su fundación ha tenido como visión convertirse en un centro de investigación líder a nivel nacional y regional (CIRA/ UNAN, 2009), lo que ha implicado elevar los niveles de calidad en los campos de docencia, investigación, generación de información básica a través de determinaciones analíticas, y la formación de especialistas con su programa de Maestría, servicios de asesoría técnica y extensión universitaria, que respondan a las expectativas de la comunidad nicaragüense. En la actualidad, la globalización demanda el desarrollo competitivo de los laboratorios de ensayo, para lo cual surge la necesidad de implementar los sistemas de gestión de la calidad que mejoren continuamente la capacidad operacional y la competencia técnica de los laboratorios, que se resume en la acreditación de los mimos. En este contexto, surge la necesidad de establecer 
e implementar un sistema de gestión de la calidad en el CIRA/UNAN, que asegure su competencia técnica, mediante el apego a la norma ISO/IEC 17025:2005 o su equivalente nacional NTN 04 001 05, por ser el mecanismo internacionalmente aceptado para la demostración de la competencia técnica de los laboratorios, (ISO, 2005).

El presente trabajo describe el proceso de implementación del Sistema de Gestión de la Calidad (SGC) en los laboratorios del CIRA/UNAN. La implementación de este sistema ha asegurado la mejora continua y el manejo más eficiente de los recursos humanos y financieros, lo que a la vez ha permitido acreditar nueve ensayos Microbiológicos e iniciar el proceso de acreditación de otros ensayos.

\section{Materiales y Métodos}

El proceso de implementación de un sistema de gestión de la calidad en los laboratorios del Centro para la Investigación en Recursos Acuáticos de Nicaragua, se desarrolló en cuatro etapas fundamentales, que consistieron en: Diagnostico situacional del Centro, Establecimiento del Sistema de Gestión de la Calidad, Implementación y Mantenimiento (figura 1).

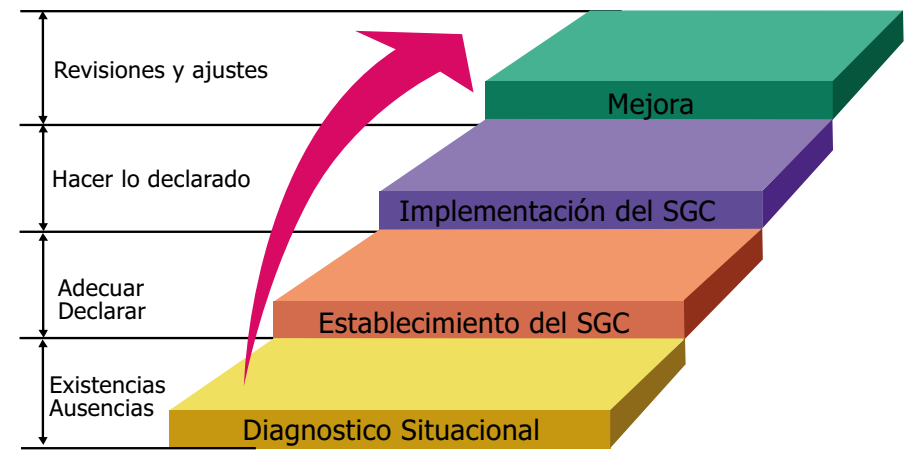

Figura 1. Etapas de implementación del Sistema de Gestión de la Calidad en el Centro para la Investigación de Recursos Acuáticos de Nicaragua (CIRA/UNAN)
Previo al establecimiento e implementación del sistema de gestión de la calidad, se realizó un diagnostico situacional del Centro, con el objeto de identificar las fortalezas y debilidades existentes en los laboratorios, tanto en recursos humanos como en infraestructura, este diagnostico se realizó a través de visitas a los diferentes laboratorios y unidades, para identificar el grado de conocimiento y compromiso que tenía el personal con el aseguramiento y control de la calidad, estado de la documentación y de los procedimientos analíticos, equipos e instalaciones físicas disponibles.

El establecimiento del sistema de gestión de la calidad, se llevo a cabo en el período comprendido de febrero a julio del año 2006. Para esto, se realizó una reunión con la dirección del centro, con el objeto de establecer e identificar las bases sobre las cuales se establecería el sistema de gestión de la calidad, acordándose que el sistema de gestión de la calidad se basaría en la norma ISO/IEC 17025:2005 o su equivalente nacional NTN 04 001-05 (ONA/ MIFIC, 2005) y en la estructura organizativa del Centro. En la figura 2, se ilustra el proceso seguido durante el establecimiento del sistema de gestión.

Dado que la Norma ISO/IEC 17025:2005 está diseñada para un laboratorio y no para una

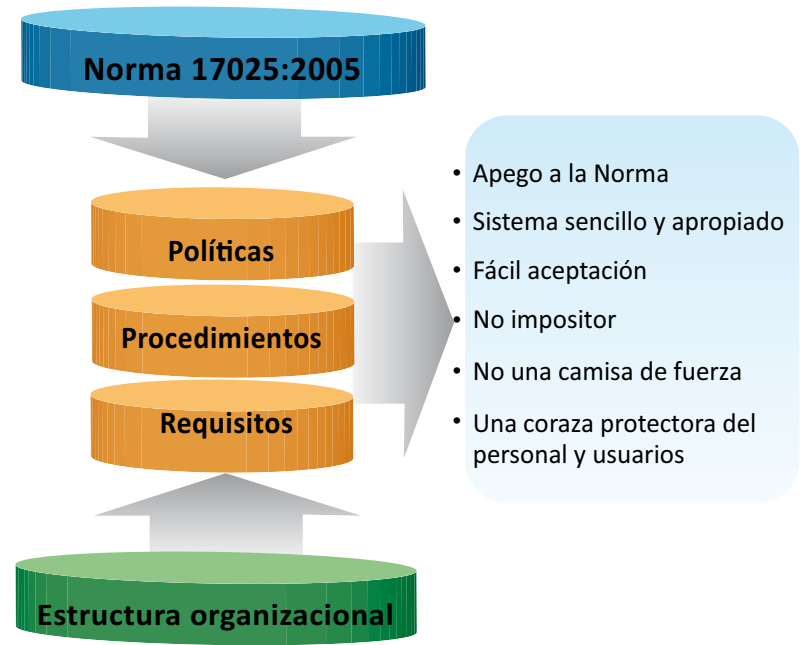

Figura 2. Diagrama del establecimiento del Sistema de Gestión de la Calidad 
organización más grande y compleja como el CIRA/ UNAN, el cual integra nueve laboratorios con diferentes disciplinas: Química, Biología Acuática, Microbiología e Hidrogeología, se decidió generar un documento único para los requisitos de gestión, y los requisitos técnicos, por la complejidad de la institución y los diferentes quehaceres operativos de cada laboratorio, fueran abordados y descritos por el personal de los diferentes laboratorios en estricto apego a la norma.

La implementación del Sistema de Gestión de la Calidad (figura 3) inició en septiembre del 2006, con las siguientes líneas estratégicas:

Divulgación del sistema de gestión, la cual se realizó, mediante la entrega de una copia de la documentación del sistema de gestión a cada laboratorio, área y unidades del centro involucradas en el sistema de gestión de la calidad, seguido de un proceso de familiarización por parte del personal con el sistema de gestión de la calidad, el cual se efectuó mediante sesiones de lectura de la documentación por el personal de laboratorio, áreas y unidades.

Elaboración de un plan de trabajo general, dirigido a la implementación del sistema, donde se programó, la implementación de cada uno de los procedimientos, que conforma el sistema de gestión.

Se estableció un calendario de visitas de seguimiento por parte del personal del Área Técnica, Aseguramiento y Control de la Calidad del Centro, durante las cuales, se brindó asesoría y orientación en la implementación del sistema, posteriormente las visitas de implementación se orientaron a monitorear el grado de avance de la implementación y mantenimiento del sistema de gestión.

El mantenimiento y mejora del sistema de gestión se aseguró a través de la realización de programas anuales de auditoría interna en cada laboratorio, para lo cual

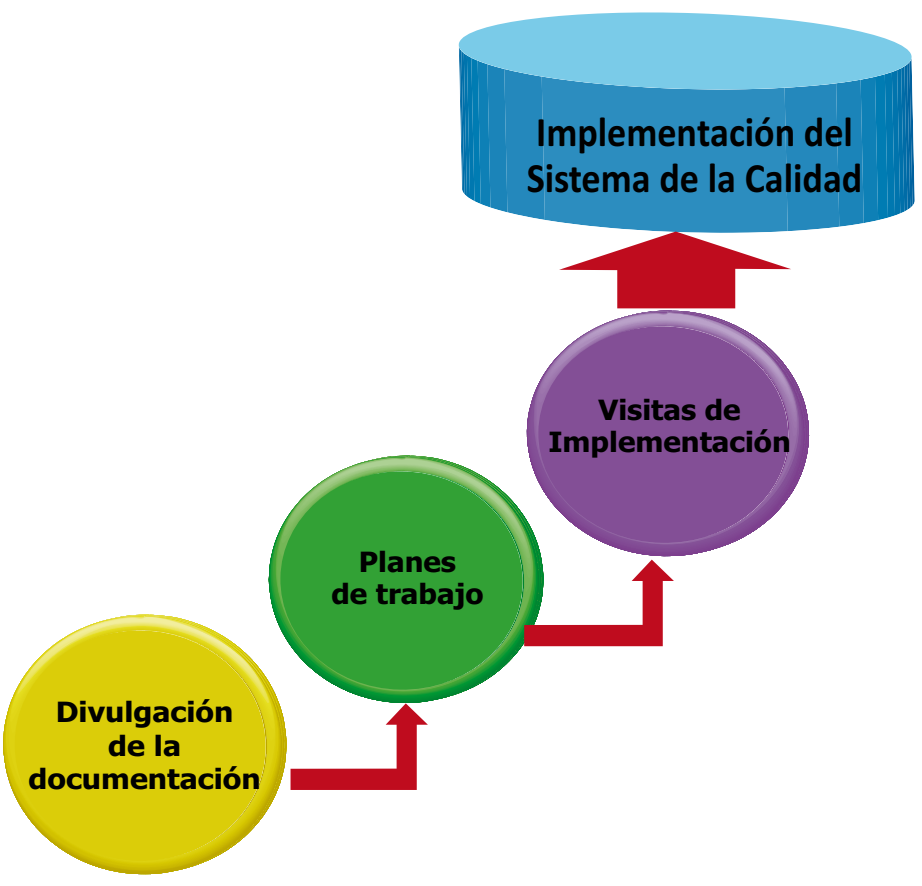

Figura 3. Diagrama de la implementación del Sistema de Gestión de la Calidad

se conformó y capacitó a un grupo de evaluadores internos de la calidad. Durante estos procesos de auditoría, se encontraron algunos hallazgos que han contribuido a la mejora del sistema de gestión de la calidad, tales como; mejoras en infraestructura, equipamiento, documentación generada por los laboratorios, adquisición de materiales de referencia empleados en el aseguramiento y control de la calidad de los análisis. A través de un proceso sistemático de supervisión por parte de los jefes de laboratorio y jefes de área se han identificado trabajos no conformes, y potenciales no conformidades, para los cuales se ha trazado acciones correctivas y acciones preventivas.

También se estableció un programas de revisión anual del sistema de gestión por parte de la dirección del Centro, durante el cual se revisa todo el sistema, políticas y objetivos de la calidad, indicadores, resultados de auditorías internas, cierre de acciones correctivas, análisis de la información de retorno de clientes, usuarios e investigadores del Centro, quejas presentadas por clientes, planes 
futuros, necesidades de capacitación del personal, participación y resultados obtenidos en ejercicios de intercomparación o ensayos de aptitud, y otros aspectos técnicos, tales como validación de métodos, necesidades de adquisión de equipos, mejora de infraestructura entre otros.

\section{Resultados y Discusión}

En el análisis situacional se detectaron las siguientes fortalezas: existencia de una cultura de la calidad, personal capacitado y comprometido con la calidad, un alto porcentaje de procedimientos analíticos documentados, implementación de controles de calidad en los análisis, existencia de equipos adecuados para el desarrollo de diferentes metodologías analíticas, instalaciones e infraestructura medianamente adecuadas, la dirección del Centro comprometida con la calidad. Como debilidades se detectó que: la mayor parte de los procedimientos no habían sido validados, faltaban algunos procedimientos operativos que aun no habían sido documentados, no todas las operaciones y actividades que se realizaban en el centro y en los laboratorios se encontraban declarados, se carecía de un Manual de la calidad y de otros documentos de importancia, que era necesario realizar mejoras en infraestructura y en equipos, tanto en mantenimiento como en calibración de los mismos.

Durante el establecimiento e implementación del Sistema de Gestión de la Calidad, resulto clave el compromiso y apoyo de la dirección del Centro, siendo las primeras acciones, el nombramiento de un técnico de experiencia como encargado de la calidad, la formación del Área Técnica Aseguramiento y Control de la Calidad, y la destinación de recursos suficientes para la implementación del sistema de gestión y para el cumplimiento de los requisitos de la Norma, tales como: i) cambio y mejora de infraestructura, ii) adquisición de nuevos equipos, compra de materiales de referencias, materiales de oficina, capacitaciones técnicas, capacitaciones en Norma 17025:2005 y iii) la formación del grupo de evaluadores del centro. El sistema de gestión de la calidad integra todos los procedimientos administrativos y operativos que establece la Norma, en el se declararon todas las políticasy procedimientos concernientes a la parte de gestión de la Norma, que corresponden a los aspectos de: organización, sistema de gestión, control de documentos, revisión de pedidos, ofertas y contratos, subcontratación de ensayos, compras de servicios y suministros, servicio al cliente, control de trabajos no conformes, mejoras, acciones correctivas y preventivas, auditorías internas, control de registros y revisiones por la dirección, todos contenidos en el Manual del Sistema de Gestión de la Calidad (CIRA/UNAN, 2009) y en el Manual de Procedimientos de Gestión de la Calidad (CIRA/UNAN, 2009).

Se generaron cuatro documentos en cada laboratorio: (1) Manual de Aseguramiento y Control de la Calidad (CIRA/UNAN, 2009) (2) Manual de Procedimientos Operativos del Aseguramiento y Control de la Calidad (CIRA/UNAN, 2009), (3) Manual de Procedimientos Operativos Normalizados (CIRA/UNAN, 2008) y (4) Manual de Uso, Mantenimiento, Calibración y Control de Equipos (CIRA/UNAN, 2008), estos documentos se interrelacionan entre si y a su vez con el manual del sistema de gestión de la Calidad del Centro.

\section{Conclusiones}

El Centro para la Investigación en Recursos Acuáticos de Nicaragua, cuenta con un sólido y completo sistema de gestión de calidad que rige todas las operaciones administrativas y técnicas asociadas a la generación de resultados, proyectos, informes e interpretaciones, lo cual lo posiciona como uno de los mejores Centros de Investigación a nivel nacional. 
E1 personal del CIRA/UNAN opera apegado al Sistema de Gestión de la Calidad y posee una cultura de calidad, la cual ha sido formada desde hace mas de dos décadas, lo que facilito el proceso de implementación del Sistema de Gestión de la Calidad.

La dirección del Centro está comprometida a cumplir con todos los requisitos de la Norma y ha establecido como requisito de ineludible cumplimiento, que todo el personal debe conocer e implementar el sistema de gestión de la calidad.

La acreditación de nueve ensayos microbiológicos, valida el sistema de gestión de la calidad del CIRA/ UNAN.

\section{Bibliografia}

CIRA/UNAN. (2008). MPON- Laboratorio-03. Manual de procedimientos operativos normalizados, ATACC-CIRA/UNAN (Documento Interno).

CIRA/UNAN. (2008). MUMCCEL- Laboratotrio-04. Manual de uso, mantenimiento, control y calibración de equipos del laboratorio, ATACC-CIRA/UNAN (Documento Interno).

CIRA/UNAN. (2009). MACCL-Laboratorio-01. Manual de Aseguramiento y Control de la Calidad del laboratorio, ATACC-CIRA/UNAN (Documento interno).

CIRA/UNAN. (2009). MPGC-CIRA-02. Manual de Procedimientos de Gestión de la Calidad, 1-140, ATACC-CIRA/UNAN (Documento interno).

CIRA/UNAN. (2009). MPOACCL-Laboratorio-02. Manual de Procedimientos Operativos del Aseguramiento y Control de la Calidad del Laboratorio, ATACC-CIRA/ UNAN (Documento Interno).

CIRA/UNAN. (2009). MSGC-CIRA-00. Manual del Sistema de Gestón de la Calidad, 1-71, ATACC-CIRA/UNAN (Documento Interno).

CIRA/UNAN. (10 de abril de 2009). Quienes somos: CIRA/UNAN. Extraído el 10 de abril de 2009 desde http://www.cira-unan.edu.ni.

ISO (2005). Requisitos Generales para la competencia de laboratorios de ensayo y calibración. Norma ISO/IEC 17025:2005, 1-29.

ONA/MIFIC. (2005). Requisitos Generales para la competencia de laboratorio de ensayo y calibración. Norma NTN 04001 05, 1-29. 